\title{
Die Rolle von Histonmethylierungen in der Entwicklung des zentralen Nervensystems und bei Neuralrohrdefekten
}

https://doi.org/10.1515/nf-2018-0001

Zusammenfassung: Es ist essenziell, die Vorgänge, die während der Entwicklung des zentralen Nervensystems (ZNS) ablaufen, zu verstehen, um Krankheitsbilder wie Mikrozephalie oder Neuralrohrdefekte, welche aus fehlerhaften Prozessen während der Entwicklung des ZNS resultieren, zu entschlüsseln.

Epigenetische Mechanismen sind spatiotemporale Regulationsmechanismen, die auf die Genexpression Einfluss nehmen. Sie sind in mannigfaltige zelluläre Vorgänge involviert, unter anderem in die Zelldifferenzierung. Folglich besteht großer Bedarf, diese Mechanismen in Hinblick auf Entwicklung und Krankheitsbilder zu erforschen. In dem vorliegenden Artikel werden wir Histonmethylierung als epigenetische Modifikation beleuchten und die Mechanismen erläutern, wie sie Genexpression und Zelldifferenzierung während der Entwicklung des zentralen Nervensystems, sowie neuraler Differenzierung beeinflussen. Ferner werden wir den Zusammenhang zwischen Histonmethylierung und der Ätiologie von Neuralrohrdefekten diskutieren. Es wird besonders auf die Rolle von disruptor of telomeric silencing like 1 (DOT1L) und Histon H3 Lysin 79-Methylierung (H3K79me) eingegangen, welche eine ungewöhnliche Histonmethylierung darstellt, jedoch relevant für die fehlerfreie Entwicklung des zentralen Nervensystems ist.

Schlüsselwörter: Entwicklung; neurale Differenzierung; Epigenetik; Histonmethylierung; Neuralrohrdefekte

\footnotetext{
*Korrespondenzautorin: Tanja Vogel, Albert-Ludwigs-Universität Freiburg, Medizinische Fakultät, Institut für Anatomie und Zellbiologie, Abteilung für Molekulare Embryologie, Albertstr. 17, 79104 Freiburg, Deutschland, E-Mail: Tanja.Vogel@anat.uni-freiburg.de Alejandro Villarreal, Henriette Franz, Albert-Ludwigs-Universität Freiburg, Medizinische Fakultät, Institut für Anatomie und Zellbiologie, Abteilung für Molekulare Embryologie, Albertstr. 17, 79104 Freiburg, Deutschland, E-Mail: Alejandro.Villarreal@anat.uni-freiburg. de, Henriette.Franz@anat.uni-freiburg.de
}

\section{Einleitung}

In einem multizellulären Organismus wie dem menschlichem Körper stammen alle Zellen von einer einzigen Zelle, der Zygote ab, die aus der Verschmelzung von Ei und Spermium hervorgeht. Nach der Befruchtung proliferiert die Zygote und es bilden sich zahlreiche andere Zellen, Gewebe und Organe - bis sich ein ganzer Organismus ergeben hat. Dieser Prozess wird Embryogenese genannt. Ein zentrales Ereignis während der Embryogenese ist die Gastrulation - die Phase, in der sich die drei Keimblätter ausbilden (Kiecker et al., 2016; Solnica-Krezel und Sepich, 2012; Tseng et al., 2017). Die Keimblätter werden als Endoderm, Mesoderm und Ektoderm bezeichnet. Im Laufe der weiteren Entwicklung geht aus dem Ektoderm das Neuralrohr hervor, aus welchem wiederum das zentrale Nervensystem entsteht (Greene und Copp, 2014; Smith und Schoenwolf, 1997) (Abbildung 1A). Das zentrale Nervensystem umfasst zwei zentrale anatomische Strukturen: das Gehirn und das Rückenmark. Diese Strukturen beinhalten unterschiedliche Arten von Neuronen, welche im Körper elektrische Signale ausschütten und empfangen. Neben den Neuronen sind Astrozyten, Oligodendroyzten und Mikroglia ebenso Zellen des zentralen Nervensystems (Abbildung 1B). Astrozyten versorgen Neurone mit Metaboliten, regulieren synaptische Übertragung, sind an der Bildung der Blut-Hirn-Schranke beteiligt und besitzen neuroprotektive Eigenschaften bei Hirnverletzungen. Oligodendrozyten bilden die Myelinscheiden, welche die neuronalen Axone umgeben. Interessanterweise haben alle Zellarten außer Mikroglia, welche aus Vorläuferzellen im Dottersack hervorgehen, einen gemeinsamen Ursprung im zentralen Nervensystem. Sie gehen allesamt aus einer gemeinsamen Vorläuferzelle hervor. Diese zunächst multipotenten Vorläuferzellen sind neurale Stammzellen, welche im Neuralrohr angesiedelt sind. Während der Entwicklung des zentralen Nervensystems erwerben diese neuralen Stammzellen die Fähigkeit zu proliferieren, um mehr Stammzellen zu generieren oder aber die Fähigkeit, 
A

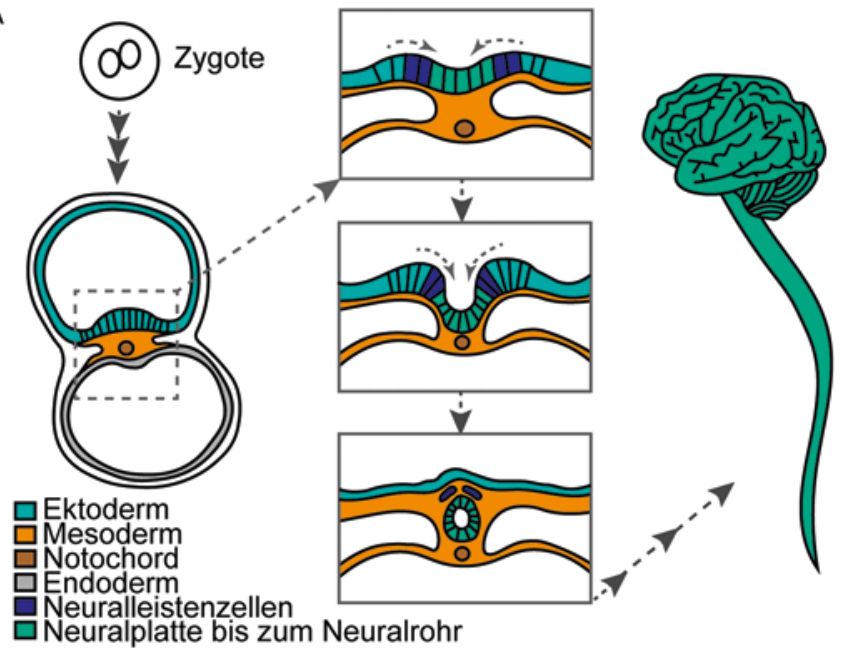

B

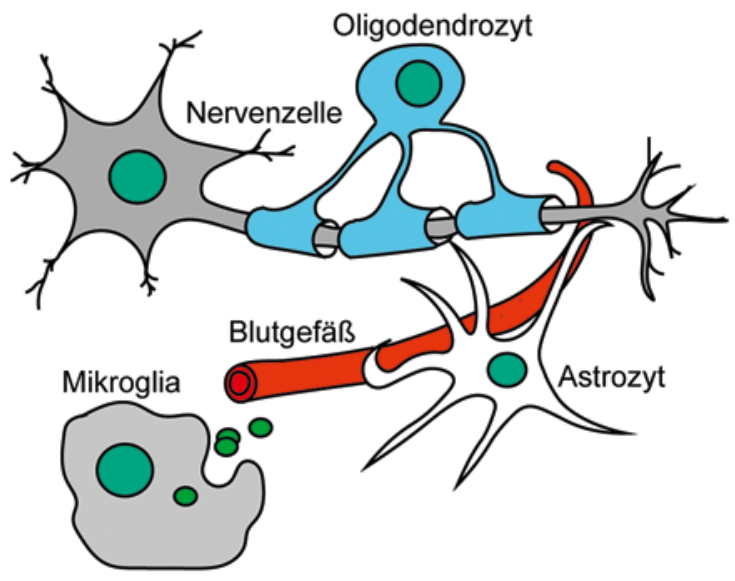

Abb. 1: A. Die Entwicklung von der Zygote über das Neuralrohr bis hin zum ZNS beim Menschen. B. Neurone, Astrozyten, Oligodendrozyten und Mikroglia sind die wichtigsten Zellarten des ZNS.

den Differenzierungsprozess zu initiieren, welcher in der Neuro-, Astro- oder Oligodendrogenese mündet. Während proliferative Zellteilung zu der Entstehung einer Tochterzellgeneration mit dem gleichen Entwicklungspotenzial führt, wird durch die differenzierende Zellteilung das Potenzial der Zelle eingeschränkt und für die Tochterzelle ein bestimmtes Zellschicksal festgelegt. Um die Proliferation aufzuhalten und die Differenzierung zu initiieren, bedarf es bestimmter Signale. Neurale Stammzellen limitieren also schrittweise ihre proliferativen Eigenschaften und lenken ihr differenzierendes Potenzial in eine bestimmte Richtung - ein Phänomen, welches als Zellschicksal bezeichnet wird (Hirabayashi und Gotoh, 2010; Qiao et al., 2016; Tang et al., 2015).

Was jedoch macht ein Neuron zu einem Neuron, einen Astrozyten zu einem Astrozyten und einen Oligodendrozyten zu einem Oligodendrozyten? Wie weiß eine Zelle, welchen Phänotyp und welche Funktionen sie annehmen muss? Wie wird ein festgelegtes Zellschicksal beibehalten und nicht wieder rückgängig gemacht? Seit Langem ist bekannt, dass das Zellschicksal und somit Zellfunktion durch die Aktivität oder Transkription bestimmter Gene bestimmt wird. Jede einzelne Zelle stammt von der Zygote ab; somit haben alle Zellen die gleiche DNA-Sequenz und Genkomposition. Die Entscheidung, zu welchem Zelltyp sich eine Zelle entwickelt, hängt davon ab, welche Gene zu einem bestimmten Zeitpunkt aktiviert werden. Es ist also von wesentlicher Bedeutung, zu verstehen, nach welchen Kriterien diese Gene ausgewählt werden und wie deren Transkription aktiviert, reprimiert oder in der Balance gehalten wird, je nachdem, welches Zellschicksal angestrebt wird. Es gibt zahlreiche und vielfältige Mechanismen, mit welchen auf die Gentranskription Einfluss genommen werden kann. In dem vorliegenden Artikel werden wir uns ausschließlich auf epigenetische Mechanismen beschränken, welche Histonmodifikationen mit einschließen und darstellen, wie diese Proteinmodifizierungen die Gentranskription im Bezug auf Zelldifferenzierung im zentralen Nervensystem beeinflussen.

\section{Die Chromatinstruktur}

Histone sind kleine Proteine, welche die Fähigkeit besitzen, DNA zu binden. Die strukturelle Einheit von Histonen und DNA wird als Chromatin bezeichnet. Jeweils zwei der Kernhistone, H2A, H2B , H3 und H4 schließen sich zu einem Oktamer zusammen. 146 bp der DNA winden sich 1,5-mal um jedes Histonoktamer. Die kleinste Vepackungseinheit des Chromatins, der DNA-Histon-komplex, wird als Nukleosom bezeichnet. Ein fünfter Histontyp befindet sich außerhalb des Nukleosoms. Dieses stellt das Verbindungshiston $\mathrm{H} 1$ dar, welches auf höherer Ebene dazu dient, DNA kompakt zu verpacken (Cutter und Hayes, 2015; Lawrence et al., 2016). Genauso wie andere zelluläre Proteine unterliegen Histone nach ihrer Synthese chemischen Modifikationen. Der Vorgang der Proteinsynthese wird als Translation bezeichnet und die darauffolgenden chemischen Veränderungen der Proteine als posttranslationale Modifikationen. Posttranslationale Modifikationen ermöglichen Proteinen beispielsweise eine veränderte enzymatische Aktivität, Interaktion mit bestimmten Proteinen oder die Lokalisierung innerhalb eines bestimmten 

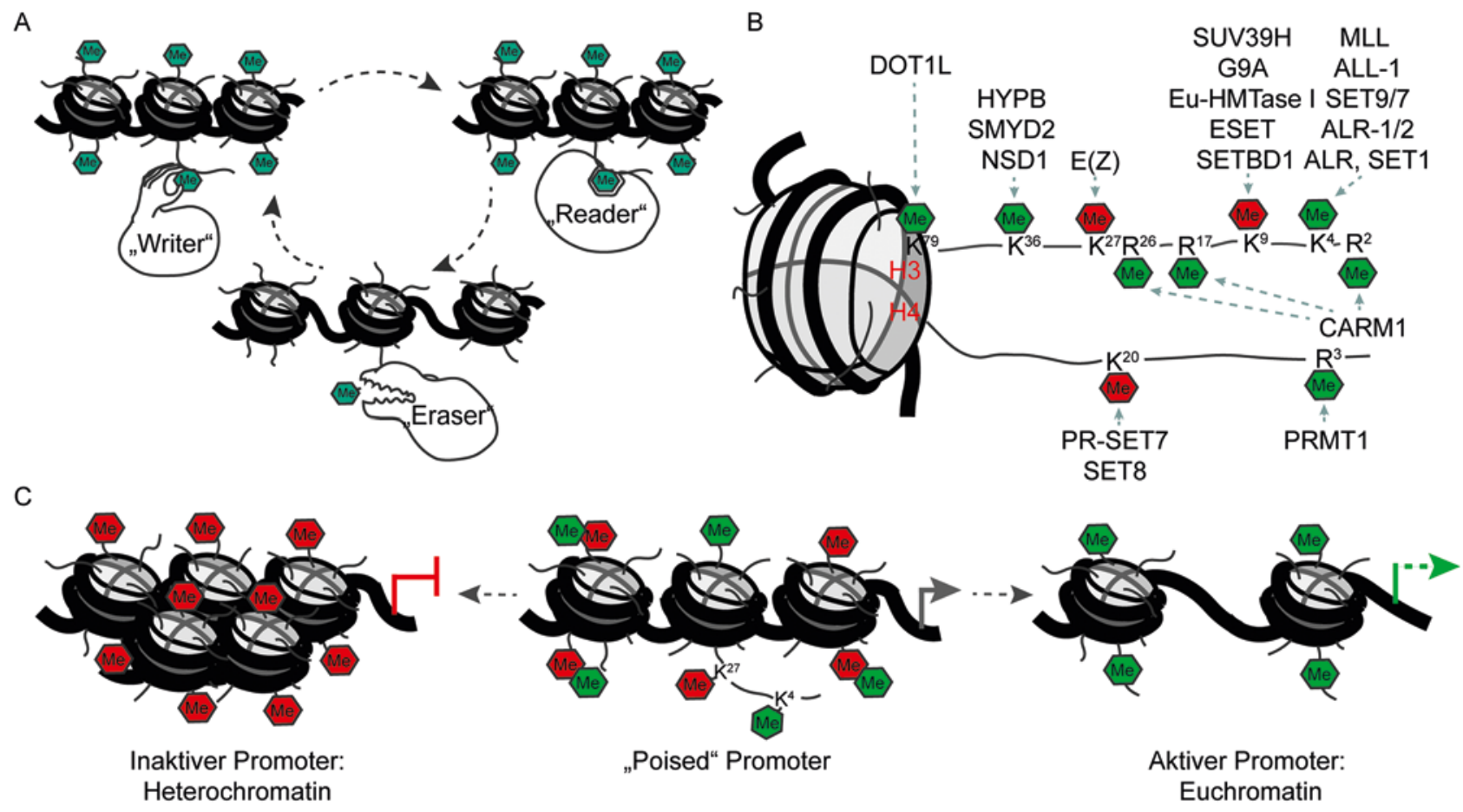

Abb. 2: A. Schreiber (writer)-, Leser (reader)- und Auslöscher (eraser)-Proteine von Histon-Modifikationen. B. Modifikatoren von HistonMethylierungen und ihre writer Enzyme. Grün: Transkriptions-Aktivierung. Rot: Transkriptions-Repression. C. Bivalente Promoter sind gleichzeitig mit aktivierenden und reprimierenden Modifikationen versehen. Während der Entwicklung einer bestimmten Zellart bleibt eine Modifikation erhalten und das entsprechende Gen wird aktiviert oder reprimiert.

zelluären Kompartments. Von diesen posttranslationalen Modifikationen wurden bislang die folgenden am besten untersucht: Acetylierung, Phosphorylierung, Methylierung, Desaminierung, Ubiquitinierung, ADP-Ribosylierung und Sumoylierung (Lawrence et al., 2016; Peserico und Simone, 2011; Prachayasittikul et al., 2017; Yang und Seto, 2007). Die posttranslationale Modifikation von Histonen zählt zu den epigenetischen Modifizierungen, da sie das Potenzial besitzen, in die nächste Zellgeneration weitergereicht $\mathrm{zu}$ werden. Zusätzlich besitzen sie bestimmte Informationen bezüglich des Transkriptionsstatus des entsprechenden genomischen Lokus. Diese Modifikationen werden durch Enzyme gesteuert, die sogenannten „Schreiber“ (writer), durch die „Leser“ (reader) Moleküle erkannt und durch „Auslöscher“ (eraser) wieder entfernt (Abbildung 2A). Somit sind posttranslationale Modifikationen sehr anpassungsfähig und stellen eine weitere Möglichkeit dar, zusätzliche vererbbare Information neben dem genetischen Code zu liefern. Unterschiedliche posttranslationale Histonmodifikationen verändern die Struktur des Chromatins, indem sie sie mehr (offenes Chromatin) oder weniger (geschlossenes Chromatin) zugänglich für die Transkriptionsmaschinerie machen. Dieser Mechanismus ist äußerst wichtig, da offenes Chromatin ge- wöhnlich Gene enthält, welche aktiv transkribiert werden, in geschlossenem Chromatin hingegen werden Gene nicht transkribiert. Offenes und aktives Chromatin wird als Euchromatin bezeichnet, geschlossenes Chromatin als Heterochromatin (Cutter und Hayes, 2015; Luger et al., 2012; Venkatesh und Workman, 2015).

\section{Regulierung der Chromatinzugäng- lichkeit durch Histonmodifikationen}

Histone können an bestimmten Aminosäuren modifiziert werden. Sowohl die Position der modifizierten Aminosäure im Histon, als auch die chemische Gruppe, welche an die Aminosäure gebunden ist, enthalten Information bezüglich der Chromatinzugänglichkeit. Posttranslationale Modifikationen haben Einfluss auf die Interaktion zwischen den positiv geladenen Histonen und den negativ geladenen Phosphatgruppen der DNA. Wird diese Interaktion z. B. durch eine Erhöhung der Negativladung an den Histonen abgeschwächt, wird das Chromatin offener und zugänglicher für Transkriptionsfaktoren. Dies kann dadurch erzielt werden, indem ein Histon beispielsweise 
acetyliert und somit die Stabilität des gesamten Nukleosoms herabgesetzt wird. Histonacetylierung wirkt folglich als Transkriptionsaktivator.

Die Auswirkungen von Histonmethylierungen auf die transkriptionale Aktivität sind jedoch um einiges vielfältiger. Abhängig von der Position der Aminosäure kann eine Methylierung entweder Aktivierung oder Ausschalten eines bestimmten Gens bewirken (Abbildung 2B). Lysin (K) kann mono-, di- oder trimethyliert werden (me1, me2 und me3), wohingegen Arginin (R) aufgrund seiner sterischen Eigenschaften nur me1 und me2 ermöglicht. Jedoch spielt nicht nur der Grad der Methylierung eine Rolle bezüglich der Genaktivität, sondern auch die Position der Aminosäure. So resultiert beispielsweise die Trimethylierung von Histon 3 an Lysin 9 oder Lysin 27 (H3K9me3 bzw. H3K27me3) in einer Genrepression. Andererseits bewirken H3K4me3 und H3K36me3 die Initiation sowie Elongation der Transkription (Lawrence et al., 2016; Peterson und Laniel, 2004). Eine weitere Variable ist die Position der Histonmethylierung innerhalb eines bestimmten Gens. So befindet sich H3K4me1 z. B. in genomischen Regionen, welche die Gentranskription erhöhen, auch bekannt als aktive Verstärker (enhancer), wohingegen sich H3K4me3 in Regionen des Genoms befindet, welche die Gentranskription initiieren, bekannt als aktive Promoter (Heintzman et al., 2007; Shlyueva et al., 2014). Das Zusammenspiel aller Histonmodifikationen innerhalb des Genoms wird als der sogenannte „Histon-Code“ bezeichnet, welcher wiederum die Transkription einzelner Gene bestimmt und somit auch das Schicksal der betroffenen Zelle.

Besonders während der Entwicklung von Zellen und Geweben befinden sich teilweise aktive und repressive Histonmodifikationen gleichzeitig an ein und demselben regulatorischen Element. Wenn beispielsweise ein solcher Histon-Code in der Promoterregion upstream der transkriptionellen Startregion angesiedelt ist, wird dieser bivalenter Promoter genannt. Grund dafür ist, dass diese Promoter, welche gleichzeitig aktivierenden H3K4me3 und dem repressiven H3K27me3 bzw. H3K9me3 aufweisen, sich in einem sogenannten bivalenten Zustand befinden (Abbildung 2C). Das downstream Gen eines bivalenten Promoters bleibt in der Regel inaktiv. Aber es bedarf lediglich der Entfernung der repressiven Histonmethylierung, um dieses Gen zu aktivieren, da die Information hierfür bereits vorhanden ist. Dieser bivalente Zustand ermöglicht somit eine rasche temporale Aktivierung der Genexpression, was eine essenzielle Voraussetzung für fehlerfreie Differenzierungsprozesse ist (Harikumar und Meshorer, 2015; Hirabayashi und Gotoh, 2010).

Weiterhin kann sich die Bildung einer bestimmten Histonmodifizierung positiv oder negativ auf die Ausbil- dung einer zweiten auswirken. Ein Beispiel hierfür ist die Ubiquitinierung von H2B, welche die Dimethylierung von H3K79 durch die Methyltransferase disruptor of teleomeric silencing-like 1 (DOT1L) (Abbildung 3A) unterstützt. Dieses Konzept wird als „Histon Crosstalk“ bezeichnet (Suganuma und Workman, 2008; Nguyen und Zhang, 2011; Zhang et al., 2015).

Für die Zelldifferenzierung wird sowohl Genrepression als auch Genaktivierung benötigt. Beide Prozesse müssen in einem minuziösen temporospatialen Zusammenspiel erfolgen, um die fehlerfreie Differenzierung einer Zelle zu gewährleisten. Viele Transkriptionsfaktoren stimmen die Expression unterschiedlicher Gene aufeinander ab, wofür sie Zugang zum Chromatin benötigen und mit enhancer oder Promoterregionen interagieren müssen. Folglich sind Histonmodifikationen wichtige upstream Regulatoren, da durch diese die Zugänglichkeit des Chromatins reguliert wird. Außer dieser Histonmodifikationen gibt es noch andere molekulare Mechanismen, wie z. B. nicht-kodierende RNA oder DNA-Methylierung, durch welche die Aktivität von Transkriptionsfaktoren sowie die Chromatinzugänglichkeit zusätzlich reguliert werden können (Imamura et al., 2014).

\section{Histonmodifikationen während der neuralen Zelldifferenzierung}

Im Laufe der Entwicklung und Differenzierung wird das Potenzial von Stammzellen kontinuierlich limitiert (Hirabayashi und Gotoh, 2010; Lomvardas und Maniatis, 2016). Wie bereits erwähnt, differenzieren neurale Stammzellen bereits früh zu Neuronen und erlangen im Laufe der späteren Entwicklung die Fähigkeit, sich zu Astrozyten zu differenzieren. Die Umlenkung des transkriptionellen Programms, was die Voraussetzung für die Differenzierung zu einem bestimmten Zelltyp ist, geht mit Chromatinmodifikationen einher. Im Detail werden Gene, welche an Differenzierungsprozessen beteiligt sind, in Abhängigkeit von den Histonmodifikationen am entsprechenden Promoter aktiviert. Ein Beispiel hierfür ist Ngn1 (Neurogenin 1), ein Gen, das ausschließlich in neuralen Stammzellen exprimiert wird, welche dazu bestimmt sind, zu Neuronen zu differenzieren (neurogene neurale Stammzellen) (Hirabayashi und Gotoh, 2010; Hirabayashi et al., 2004). In der embryonalen Stammzelle ist der Promoter von Ngn1 mit repressiven und aktivierenden Modifikationen ausgestattet (H3K27me3 bzw. H3K4me3). Die repressive Modifizierung H3K9me3 ist in diesem Stadium ebenfalls am Ngn1 Promoter vorhanden. Während der Differenzierung ändert 
A

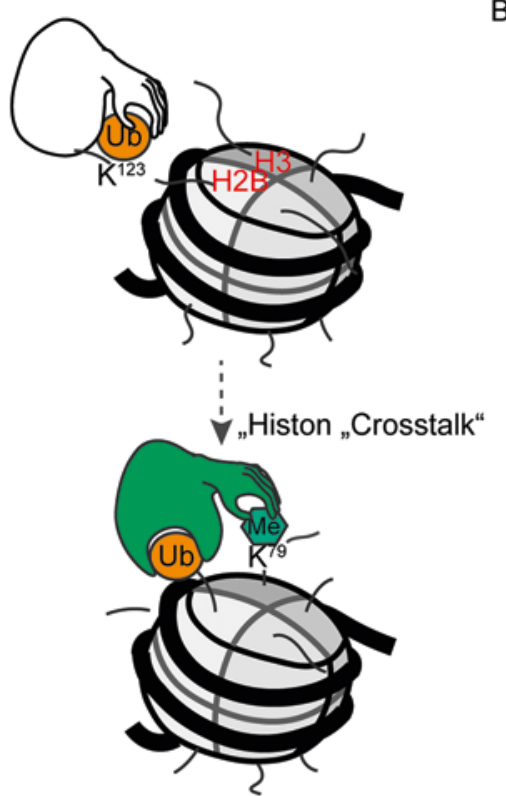

C

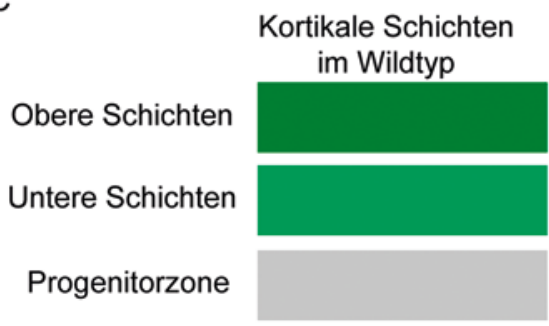

B
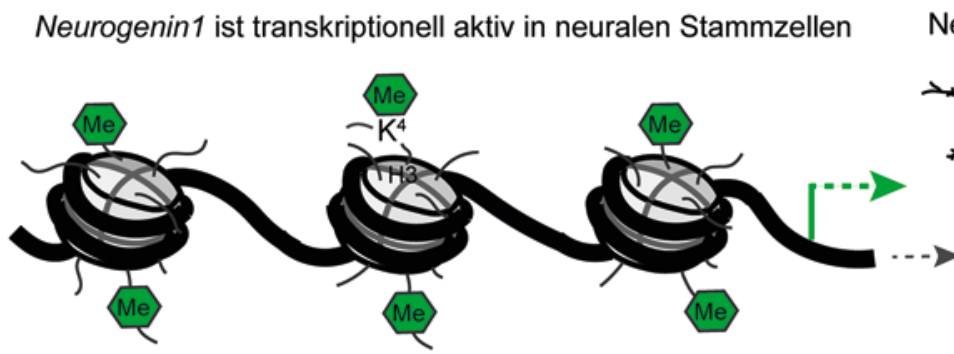

Neurogenin1 ist transkriptionell inaktiv in neuralen Stammzellen
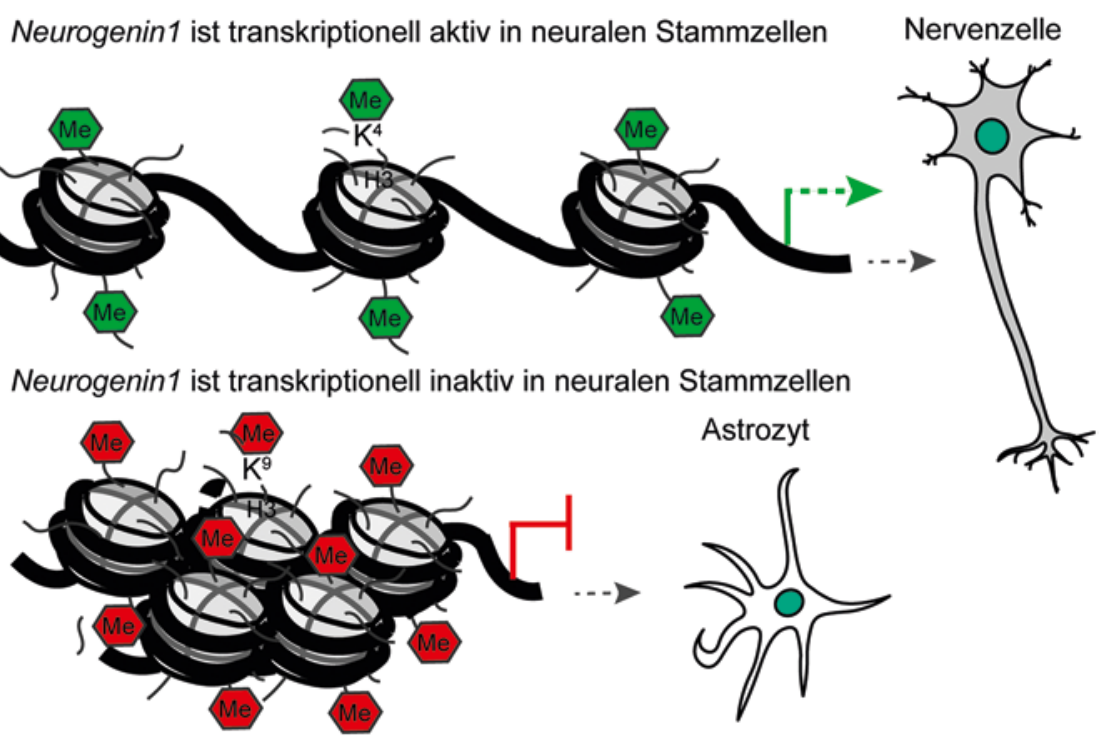

Kortikale Schichten in DOT1L-CKO/AF9-cKO

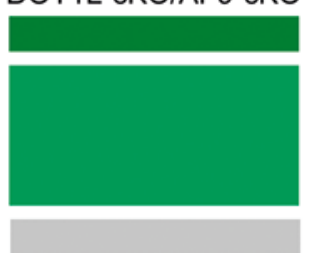

Abb. 3: A. Beispiel des Histon-Crosstalks: die Ubiquitinierung von H2B Lysin 123 (H2Bub) ermöglicht die Methylierung von H3 Lysin 79 (H3K79). B. Das Schicksal einer neuralen Stammzelle (NSC, neural stem cell) wird durch die Modifikation des Ngn1 Promoter und dessen Expression bestimmt. Die Methylierung von H3 Lysin 4 (H3K4) am Ngn1 Promoter in NSCs führt zur Expression des Ngn1 Gens und legt somit ein neuronales Zellschicksal fest. Erfolgt die Methylierung jedoch an H3 Lysin 9 (H3K9), hat dies die Repression von Ngn1 zur Folge und führt zum Zellschicksal eines Astrozyten. C. Knockout von Af9 oder Dot1l führt zur Bildung von Neuronen, die Marker exprimieren, welche charakteristisch für die unteren Schichten im zerebralen Kortex sind. Die Anzahl der Neuronen in den oberen Schichten und neuraler Stammzellen in der Vorläufer Zone wird dadurch verringert.

sich dieser bivalente Zustand des Promoters, indem die repressiven Modifikationen entfernt werden. In diesem Falle differenziert die embryonale Stammzelle zu einem Neuron, in welchem Ngn1 aktiv ist (Hirabayashi und Gotoh, 2010; Mikkelsen et al., 2007). Im Gegensatz dazu differenziert die neurale Stammzelle zu einem Astrozyten, wenn Ngn1 reprimiert bleibt (Abbildung 3B). Die epigenetische Regulierung von Ngn1 stellt ein gutes Beispiel dafür dar, wie ein bivalenter Promoter einen aktivierenden oder repressiven Zustand annehmen kann, was wiederum das Schicksal der betroffenen Zelle bestimmt (Neuron oder Astrozyt).

Ein weiteres Beispiel ist der Promoter des Gfap Gens, welches für GFAP (glial fibrillary acidic protein) in Astrozyten kodiert. Während der Differenzierung von Astrozyten wird Gfap aktiviert, wohingegen es in Neuronen inaktiv ist. Die Aktivierung von Gfap erfolgt durch den Austausch des repressiven H3K9me3 am Gfap Promoter durch das aktivierende H3K4me3 ersetzt (Song und Ghosh, 2004; Takouda et al., 2017).

\section{Die Rolle von Histonmethylierungen für Funktion und Erkrankungen des zentralen Nervensystems}

Es ist durch zahlreiche Studien belegt, dass Histonmethylierungen eine wichtige Rolle in der Entwicklung und Funktion des zentralen Nervensystems spielen. So wurde beispielsweise durch post mortem Studien an menschlichen Gehirnen gezeigt, dass Mutationen in Chromatin- 
Lesern wie euchromatic histone lysine methyltranferase 1 (EHMT1), einer H3K9-Methyltransferase und lysin demethylase 5C (KDM5C), einer H3K4-Demethylase, mit geistiger Behinderung in Verbindung gebracht werden (Akbarian und Huang, 2009). Der Promoter der Glutamatrezeptoruntereinheit2 (NMDA2), essenziell für exzitatorische Synapsen, weist in den Gehirnregionen bzw. während den Entwicklungsphasen, in denen dieses Gen aktiv ist, die aktivierenden Modifikationen H3K4me2/ me3, jedoch nicht die repressive H3K27me3 auf. Während der Aktivität von Neuronen sowie Entwicklungsprozessen kommen also Histonmethylierungen zum Einsatz, um die Genexpression in einer regionspezifischen Art und Weise zu regulieren (Stadler et al., 2005). Weiterhin ergaben Untersuchungen an humanen Frontallappen, dass die Konzentration von H3K4me3 in Schizophrenie-Patienten reduziert ist (Huang et al., 2007). Rückenmarkspathologien, wie z.B. Neuralrohrdefekte, entstehen durch fehlerhafte Vorgänge während des Entwicklungsstadiums, in dem sich das Neuralrohr schließt (Greene und Copp, 2014). Beim Menschen kann die Exposition der Mutter gegenüber Substanzen, die epigenetische Mechanismen beeinflussen können, zu Neuralrohrdefekten und Verhaltensstörungen in der nachfolgenden Generation führen (Banik et al., 2017). So wird beispielsweise Folsäure (Vitamin B9) Frauen routinemäßig während der Schwangerschaft verabreicht, um so das Risiko für Neuralrohrdefekte beim Kind zu senken (Bhargava und Tyagi, 2014; Blom, 2009). Folsäure wird für die Umwandlung von Homozystein zu Methionin benötigt, welches weiter zu S-AdenosylMethionin (SAM) methyliert werden kann. SAM stellt die Methylgruppen für Methylierungsreaktionen zur Verfügung, einschließlich derer, die für Histonmethylierungen benötigt werden. Somit kann eine geringe Konzentration an Folsäure die Reduktion von SAM zur Folge haben und dadurch eine Hypomethylierung von Histonen bewirken. Wie bereits erläutert, bedarf es einer korrekten Histonmethylierung, um die fehlerfreie Transkription zu unterschiedlichen Stadien der Zellentwicklung sicherzustellen. In der Tat häufen sich die Hinweise auf eine Korrelation zwischen Neuralrohrdefekten und fehlerhafter Histonmethylierung wie z.B. H3K79me (Wilde et al., 2014; Zhang et al., 2013a). Der Gehalt an H3K79me ist in Patienten mit Neuralrohrdefekten signifikant niedriger als in gesunden Probanden (Zhang et al., 2013b). Diese Studien, kombiniert mit unseren Beobachtungen, dass DOT1L an neuronaler Differenzierung beteiligt ist, machen DOT1L und H3K79me zu neuen, wertvollen Kandidaten, um neuartige und effektive Therapiemöglichkeiten für Neuralrohrdefekte zu entwickeln. Im nachfolgenden Abschnitt werden wir den neuesten Stand der Forschung darüber erläutern, wie
DOT1L und H3K79me auf die Entwicklung des zentralen Nervensystems Einfluss nehmen.

\section{DOT1L und H3K79 - Methylierung}

Im Gegensatz zu den oben beschriebenen Methylierungen ist H3K79me nicht am Histonschwanz, der aus dem Nukleosom herausragt, lokalisiert, sondern befindet sich in der globulären Domäne vonn H3 (Abbildung 2B). H3K79me wird durch DOTL1 mono-, di- und trimethyliert. H3K79me1, me2 und me3 sind zu unterschiedlichen Anteilen in der kodierenden Region des Genoms verteilt. H3K79me1 ist hauptsächlich im mittleren Bereicht eines Gens zu finden, während sich H3K79me2/3 mehr auf die Startregion der Transkription (transcription starting site, TSS) beschränken (Deshpande et al., 2012, 2014). H3K79me2 ist in Bereichen lokalisiert, in denen aktive Transkription stattfindet (Nguyen und Zhang, 2011; Vlaming und van Leeuwen, 2016). Jedoch wird nicht jedes transkriptionell aktives Gen durch H3K79me2 markiert. Zusätzlich zeigten wir und andere Gruppen, dass die transkriptionelle Aktivität von bestimmten Genen wie z. B. Tbr1 (T Box Brain 1) (Büttner et al., 2010), ER Stress-Gene im Gehirn (Roidl et al., 2016) oder ENaCa (Scnn1a, sodium channel, nonvoltage-gated 1 alpha) in der Niere (Zhang et al., 2006) mit einer geringeren H3K79me2 einhergeht. Die Information von H3K79me scheint also spezifisch für ein bestimmtes Gen zu sein, abhängig vom Grad der Methylierung und der Lage innerhalb des Gens selbst. Es ist bemerkenswert, dass bislang weder eraser noch reader für H3K79me bekannt sind, was die genaue Funktion dieser Modifizierung weiterhin im Unklaren lässt.

Es wurde gezeigt, dass DOT1L für die Differenzierung von Kardiomyozyten sowie für die Entwicklung des Herzens, Hämatopoese und Knochenentwicklung benötigt wird (Cattaneo et al., 2016; Monteagudo et al., 2017). DOTL1 ist essenziell für die Differenzierung von Stammzellen, zum Beispiel zu Neuronen; jedoch wird es nicht für die Erhaltung der Pluripotenz von Stammzellen benötigt (Barry et al., 2009). Jüngste Studien zeigten, dass DOT1L eine Rolle bei Alterungsprozessen der Zelle spielt (obgleich die genauen Mechanismen noch nicht erforscht sind) und DOT1L-Inhibierung die Zellreprogrammierung unterstützt (Onder et al., 2012; Soria-Valles et al., 2015a, 2015b). Daten aus unserer Gruppe konnten zeigen, dass DOT1L und H3K79me die Proliferation von neuralen Stammzellen aus dem zerebralen Kortex begünstigen und dass aktives DOT1L die Transkription von ER (endoplasmatisches Retikulum) Stress-Genen in vitro unterdrückt (Roidl et 
al., 2016). In vivo beeinflusst DOT1L das transkriptionelle Programm, das für die Differenzierung von bestimmten Unterarten von Neuronen benötigt wird. Diese sind in unterschiedlichen neuronalen Schichten des zerebralen Kortex zu finden und werden unterschiedlichen Zeitpunkten der Entwicklung gebildet (Büttner et al., 2010; Franz et al., in Revision) (Abbildung 3C). Wie bereits erwähnt, wird DOT1L für die Differenzierung unterschiedlicher Zelltypen in diversen Organen benötigt. Der genaue Mechanismus jedoch, über den die H3K79me-Modifizierung von der Transkriptionsmaschinerie interpretiert wird, wird weiterhin diskutiert und ist bislang nicht geklärt (Vlaming und van Leeuwen, 2016).

\section{Die Rekrutierung von DOT1L zum Chromatin durch Chromatin reader}

DOT1L interagiert mit anderen Proteinen, um Zugang zu bestimmten Regionen des Genoms zu bekommen. So interagiert DOT1L zum Beispiel mit der RNA-Polymerase II, was den erhöhten Gehalt an H3K79me nahe der TSS oder die Verteilung von H3K79me über die gesamte kodierende Region erklären könnte (Kim et al., 2012). Mohan et al., 2010 zeigten, dass DOT1L einen Proteinkomplex bildet, der als DotCom bezeichnet wird. Dieser Komplex beinhaltet zwei Interaktionspartner von DOT1L, zum einen Myeloid/Lymphoid oder mixed-lineage leukemia 3 (MLLT3/AF9) und zum anderen 10 (MLLT10/AF10), welche in der Lage sind, unterschiedliche Chromatinzustände zu „lesen“. AF9 bindet acetylierte Histone (Li et al., 2014), wohingegen AF10 nicht-modifiziertes H3K27 bindet (Chen et al., 2015). Somit werden AF9 und AF10 zu bestimmten genomischen Regionen rekrutiert, an denen dann das entsprechende Chromatin durch deren Interaktion mit DOT1L an H3K79 methyliert wird. Dieses Zusammenspiel zwischen acetylierten und H3K79me-Regionen ist ein wichtiger Mechanismus im Krankheitsbild der Leukämie, wo Fusionsproteine von MLL und AF9 oder AF10 die Rekrutierung von DOT1L an Gene ermöglichen, welche Tumorentwicklung und -wachstum bewirken (Chen et al., 2013; Deshpande et al., 2012, 2014; Kuntimaddi et al., 2015). Wir berichteten bereits, dass AF9 DOT1L zum Chromatin im zerebralen Kortex rekrutiert und dass die AF9-DOT1L Interaktion für die Repression der Expression von TBR1 im sich entwickelnden Gehirn verantwortlich ist (Büttner et al., 2010). Indem DOT1L die Expression von TBR1 reprimiert, legt es möglicherweise das Zellschicksal eines Neurons als das eines der oberen Schicht fest (Büttner et al., 2010; Franz et al., in Revision).
Es gibt noch weitere Beispiele für das Zusammenspiel von H3K79me und anderen Histonmodifikationen. Die Ubiquitinierung von Histon 2B (H2Bub) ermöglicht die Methylierung von H3K79 durch DOT1L (Mc Ginty et al., 2008; Mohan et al., 2010; Wang et al., 2013). Diese Rekrutierung von DOT1L wurde bislang noch nicht an Neuronen untersucht, die sich gerade in der Differenzierung befinden, obgleich wir zeigen konnten, dass H2Bub für die Differenzierung von neuralen Stammzellen benötigt wird. Interessanterweise bedarf es ebenso der UbiquitinLigase RNF40, die an H2Bub beteiligt ist, um den bivalenten Zustand (H3K4me3/H3K27me3) von Genen neuronaler Abstammung aufzuheben und deren Aktivierung zu initiieren (H3K4me3) (Karpiuk et al., 2012).

\section{Zusammenfassung}

Histonmethylierungen spielen eine zentrale Rolle in der neuralen Differenzierung und der Entwicklung des zentralen Nervensystems. Die Embryogenese ist ein äußerst komplexer Prozess und auf vielfältige Weise anfällig für Störungen. Somit kann alles, was Einfluss auf diese epigenetischen Mechanismen hat, wie z. B. Mutationen in Genen, welche Chromatinmodifizierer, reader und eraser kodieren, eine fehlerfreie Entwicklung stören. Dies gilt nicht nur für die Entwicklung des zentralen Nervensystems, da die zentrale Bedeutung der Umstrukturierung des Chromatins auch für die Differenzierung anderer Gewebe beschrieben wurde (Ghayor und Weber, 2016; Martinez et al., 2015). Aufgrund dieser Entdeckungen werden derzeit weitergehende Methoden und Ansätze erforscht, mit welchen Chromatinmodifizierungen und Mechanismen der Chromatinumstrukturierung im Detail untersucht werden können (Stricker et al., 2017).

Ein detailliertes Verständnis der Mechanismen, mit denen diese Histonmodifikationen Entwicklungsprozesse beeinflussen, ist nicht nur nötig, um das allgemeine Prinzip der Genexpressionskontrolle zu verstehen, sondern ist auch eine vielversprechende Basis für die Entwicklung neuer Arzneimittel. Zusätzlich birgt es großes Potenzial für die Entwicklung von präventiven Strategien und Therapien für verschiedene Pathologien, einschließlich neurologischen und psychiatrischen Krankheitsbildern.

Danksagung: Wir danken SFB992 Medical Epigenetics, finanziert von der Deutschen Forschungsgemeinschaft, für die Unterstützung von TV (A03), sowie IBRO (International Brain Research Organization), dem DAAD (Deutscher Akademischer Austausch Dienst) und dem argentinischen 
Bildungsministerium für die postdoktoralen Stipendien, welche $\mathrm{AV}$ verliehen wurden.

Übersetzung: Dr. Joo-Hee Wälzlein

\section{Literatur}

Akbarian, S. and Huang, H.-S. (2009). Epigenetic Regulation in Human Brain - Focus on Histone Lysine Methylation. Biol. Psychiatry 65, 198-203.

Banik, A., Kandilya, D., Ramya, S., Stünkel, W., Chong, Y. S. and Dheen, S. T. (2017). Maternal Factors that Induce Epigenetic Changes Contribute to Neurological Disorders in Offspring. Genes 8.

Barry, E. R., Krueger, W., Jakuba, C. M., Veilleux, E., Ambrosi, D. J., Nelson, C. E. and Rasmussen, T. P. (2009). ES cell cycle progression and differentiation require the action of the histone methyltransferase Dot1L. Stem Cells (Dayt. Ohio) 27, 1538-1547.

Bhargava, S., and Tyagi, S. C. (2014). Nutriepigenetic regulation by folate-homocysteine-methionine axis: a review. Mol. Cell Biochem. 387, 55-61.

Blom, H. J. (2009). Folic acid, methylation and neural tube closure in humans. Birth Defects Res. Part A-Clin. Mol. Teratol. 85, 295-302.

Büttner, N., Johnsen, S. A., Kügler, S. and Vogel, T. (2010). Af9/Mllt3 interferes with Tbr1 expression through epigenetic modification of histone H3K79 during development of the cerebral cortex. Proc. Natl. Acad. Sci. U.S.A. 107, 7042-7047.

Cattaneo, P., Kunderfranco, P., Greco, C., Guffanti, A., Stirparo, G. G., Rusconi, F., Rizzi, R., Di Pasquale, E., Locatelli, S. L., Latronico, M. V. G. et al. (2016). DOT1L-mediated H3K79me2 modification critically regulates gene expression during cardiomyocyte differentiation. Cell Death Differ. 23, 555-564.

Chen, L., Deshpande, A., Banka, D., Bernt, K. M., Dias, S., Buske, C., Olhava, E. J., Daigle, S. R., Richon, V. M., Pollock, R. M. et al. (2013). Abrogation of MLL-AF10 and CALM-AF10 mediated transformation through genetic inactivation or pharmacological inhibition of the H3K79 methyltransferase Dot1l. Leukemia 27, 813-822.

Chen, S., Yang, Z., Wilkinson, A. W., Deshpande, A. J., Sidoli, S., Krajewski, K., Strahl, B. D., Garcia, B. A., Armstrong, S. A., Patel, D. J. et al. (2015). The PZP Domain of AF10 Senses Unmodified H3K27 to Regulate DOT1L-Mediated Methylation of H3K79. Mol. Cell 60, 319-327.

Cutter, A. R. and Hayes, J. J. (2015). A brief review of nucleosome structure. FEBS Lett. 589, 2914-2922.

Deshpande, A. J., Bradner, J., and Armstrong, S. A. (2012). Chromatin modifications as therapeutic targets in MLL-rearranged Leukemia. Trends Immunol. 33, 563-570.

Deshpande, A. J., Deshpande, A., Sinha, A. U., Chen, L., Chang, J., Cihan, A., Fazio, M., Chen, C.-W., Zhu, N., Koche, R. et al. (2014). AF10 regulates progressive $\mathrm{H} 3 \mathrm{~K} 79$ methylation and HOX gene expression in diverse AML subtypes. Cancer Cell 26 , 896-908.
Ghayor, C. and Weber, F. E. (2016). Epigenetic Regulation of Bone Remodeling and Its Impacts in Osteoporosis. Int. J. Mol. Sci. 17.

Greene, N. D. E. and Copp, A. J. (2014). Neural tube defects. Annu. Rev. Neurosci. 37, 221-242.

Harikumar, A. and Meshorer, E. (2015). Chromatin remodeling and bivalent histone modifications in embryonic stem cells. EMBO Rep. 16, 1609-1619.

Heintzman, N. D., Stuart, R. K., Hon, G., Fu, Y., Ching, C. W., Hawkins, R. D., Barrera, L. O., Van Calcar, S., Qu, C., Ching, K. A. et al. (2007). Distinct and predictive chromatin signatures of transcriptional promoters and enhancers in the human genome. Nat. Genet. 39, 311-318.

Hirabayashi, Y. and Gotoh, Y. (2010). Epigenetic control of neural precursor cell fate during development. Nat. Rev. Neurosci. 11, 377-388.

Hirabayashi, Y., Itoh, Y., Tabata, H., Nakajima, K., Akiyama, T., Masuyama, N. and Gotoh, Y. (2004). The Wnt/beta-catenin pathway directs neuronal differentiation of cortical neural precursor cells. Dev. Camb. Engl. 131, 2791-2801.

Huang, H.-S., Matevossian, A., Whittle, C., Kim, S. Y., Schumacher, A., Baker, S. P. and Akbarian, S. (2007). Prefrontal Dysfunction in Schizophrenia Involves Mixed-Lineage Leukemia 1-Regulated Histone Methylation at GABAergic Gene Promoters. J. Neurosci. 27, 11254-11262.

Imamura, T., Uesaka, M. and Nakashima, K. (2014). Epigenetic setting and reprogramming for neural cell fate determination and differentiation. Philos. Trans. R. Soc. B Biol. Sci. 369.

Karpiuk, O., Najafova, Z., Kramer, F., Hennion, M., Galonska, C., König, A., Snaidero, N., Vogel, T., Shchebet, A., Begus-Nahrmann, Y. et al. (2012). The Histone H2B Monoubiquitination Regulatory Pathway Is Required for Differentiation of Multipotent Stem Cells. Mol. Cell 46, 705-713.

Kiecker, C., Bates, T. and Bell, E. (2016). Molecular specification of germ layers in vertebrate embryos. Cell. Mol. Life Sci. CMLS 73, 923-947.

Kim, S.-K., Jung, I., Lee, H., Kang, K., Kim, M., Jeong, K., Kwon, C. S., Han, Y.-M., Kim, Y. S., Kim, D. et al. (2012). Human histone H3K79 methyltransferase DOT1L protein [corrected] binds actively transcribing RNA polymerase II to regulate gene expression. J. Biol. Chem. 287, 39698-39709.

Kuntimaddi, A., Achille, N. J., Thorpe, J., Lokken, A. A., Singh, R., Hemenway, C. S., Adli, M., Zeleznik-Le, N. J. and Bushweller, J. H. (2015). Degree of Recruitment of DOT1L to MLL-AF9 Defines Level of H3K79 Di- and Tri-methylation on Target Genes and Transformation Potential. Cell Rep. 11, 808-820.

Lawrence, M., Daujat, S. and Schneider, R. (2016). Lateral Thinking: How Histone Modifications Regulate Gene Expression. Trends Genet. TIG 32, 42-56.

Li, Y., Wen, H., Xi, Y., Tanaka, K., Wang, H., Peng, D., Ren, Y., Jin, Q., Dent, S. Y. R., Li, W. et al. (2014). AF9 YEATS Domain Links Histone Acetylation to DOT1L-Mediated H3K79 Methylation. Cell 159, 558-571.

Lomvardas, S. and Maniatis, T. (2016). Histone and DNA Modifications as Regulators of Neuronal Development and Function. Cold Spring Harb. Perspect. Biol. 8.

Luger, K., Dechassa, M. L. and Tremethick, D. J. (2012). New insights into nucleosome and chromatin structure: an ordered state or a disordered affair? Nat. Rev. Mol. Cell Biol. 13, 436-447. 
Martinez, S. R., Gay, M. S. and Zhang, L. (2015). Epigenetic mechanisms in heart development and disease. Drug Discov. Today 20, 799-811.

McGinty, R. K., Kim, J., Chatterjee, C., Roeder, R. G. and Muir, T. W. (2008). Chemically ubiquitylated histone H2B stimulates hDot1L-mediated intranucleosomal methylation. Nature 453, 812-816.

Mikkelsen, T. S., Ku, M., Jaffe, D. B., Issac, B., Lieberman, E., Giannoukos, G., Alvarez, P., Brockman, W., Kim, T.-K., Koche, R. P. et al. (2007). Genome-wide maps of chromatin state in pluripotent and lineage-committed cells. Nature 448, 553.

Mohan, M., Herz, H.-M., Takahashi, Y.-H., Lin, C., Lai, K. C., Zhang, Y., Washburn, M. P., Florens, L. and Shilatifard, A. (2010). Linking H3K79 trimethylation to Wnt signaling through a novel Dot1-containing complex (DotCom). Genes Dev. 24, 574-589.

Monteagudo, S., Cornelis, F. M. F., Aznar-Lopez, C., Yibmantasiri, P., Guns, L.-A., Carmeliet, P., Cailotto, F. and Lories, R. J. (2017). DOT1L safeguards cartilage homeostasis and protects against osteoarthritis. Nat. Commun. 8, 15889.

Nguyen, A. T. and Zhang, Y. (2011). The diverse functions of Dot1 and H3K79 methylation. Genes Dev. 25, 1345-1358.

Onder, T. T., Kara, N., Cherry, A., Sinha, A. U., Zhu, N., Bernt, K. M., Cahan, P., Marcarci, B. O., Unternaehrer, J., Gupta, P. B. et al. (2012). Chromatin-modifying enzymes as modulators of reprogramming. Nature 483, 598-602.

Peserico, A., and Simone, C. (2011). Physical and Functional HAT/ HDAC Interplay Regulates Protein Acetylation Balance. J. Biomed. Biotechnol. 2011.

Peterson, C. L. and Laniel, M.-A. (2004). Histones and histone modifications. Curr. Biol. 14, R546-R551.

Prachayasittikul, V., Prathipati, P., Pratiwi, R., Phanus-Umporn, C., Malik, A. A., Schaduangrat, N., Seenprachawong, K., Wongchitrat, P., Supokawej, A., Prachayasittikul, V. et al. (2017). Exploring the epigenetic drug discovery landscape. Expert Opin. Drug Discov. 12, 345-362.

Qiao, Y., Yang, X. and Jing, N. (2016). Epigenetic regulation of early neural fate commitment. Cell. Mol. Life Sci. 73, 1399-1411.

Roidl, D., Hellbach, N., Bovio, P. P., Villarreal, A., Heidrich, S., Nestel, S., Grüning, B. A., Boenisch, U. and Vogel, T. (2016). DOT1L Activity Promotes Proliferation and Protects Cortical Neural Stem Cells from Activation of ATF4-DDIT3-Mediated ER Stress In Vitro. Stem Cells (Dayt. Ohio) 34, 233-245.

Shlyueva, D., Stampfel, G. and Stark, A. (2014). Transcriptional enhancers: from properties to genome-wide predictions. Nat. Rev. Genet. 15, 272-286.

Smith, J. L. and Schoenwolf, G. C. (1997). Neurulation: coming to closure. Trends Neurosci. 20, 510-517.

Solnica-Krezel, L. and Sepich, D. S. (2012). Gastrulation: making and shaping germ layers. Annu. Rev. Cell Dev. Biol. 28, 687-717.

Song, M.-R. and Ghosh, A. (2004). FGF2-induced chromatin remodeling regulates CNTF-mediated gene expression and astrocyte differentiation. Nat. Neurosci. 7, 229-235.

Soria-Valles, C., Osorio, F. G., Gutiérrez-Fernández, A., De Los Angeles, A., Bueno, C., Menéndez, P., Martín-Subero, J. I., Daley, G. Q., Freije, J. M. P. and López-Otín, C. (2015a). NF-кB activation impairs somatic cell reprogramming in ageing. Nat. Cell Biol. 17, 1004-1013.
Soria-Valles, C., Osorio, F. G. and López-Otín, C. (2015b). Reprogramming aging through DOT1L inhibition. Cell Cycle 14, 3345-3346.

Stadler, F., Kolb, G., Rubusch, L., Baker, S. P., Jones, E. G. and Akbarian, S. (2005). Histone methylation at gene promoters is associated with developmental regulation and region-specific expression of ionotropic and metabotropic glutamate receptors in human brain. J. Neurochem. 94, 324-336.

Stricker, S. H., Köferle, A. and Beck, S. (2017). From profiles to function in epigenomics. Nat. Rev. Genet. 18, 51-66.

Suganuma, T. and Workman, J. L. (2008). Crosstalk among Histone Modifications. Cell 135, 604-607.

Takouda, J., Katada, S. and Nakashima, K. (2017). Emerging mechanisms underlying astrogenesis in the developing mammalian brain. Proc. Jpn. Acad. Ser. B Phys. Biol. Sci. 93, 386-398.

Tang, K., Peng, G., Qiao, Y., Song, L. and Jing, N. (2015). Intrinsic regulations in neural fate commitment. Dev. Growth Differ. 57, 109-120.

Tseng, W.-C., Munisha, M., Gutierrez, J. B. and Dougan, S. T. (2017). Establishment of the Vertebrate Germ Layers. Adv. Exp. Med. Biol. 953, 307-381.

Venkatesh, S. and Workman, J. L. (2015). Histone exchange, chromatin structure and the regulation of transcription. Nat. Rev. Mol. Cell Biol. 16, 178-189.

Vlaming, H. and van Leeuwen, F. (2016). The upstreams and downstreams of H3K79 methylation by DOT1L. Chromosoma.

Wang, E., Kawaoka, S., Yu, M., Shi, J., Ni, T., Yang, W., Zhu, J., Roeder, R. G. and Vakoc, C. R. (2013). Histone H2B ubiquitin ligase RNF20 is required for MLL-rearranged leukemia. Proc. Natl. Acad. Sci. U.S.A. 110, 3901-3906.

Wilde, J. J., Petersen, J. R. and Niswander, L. (2014). Genetic, epigenetic, and environmental contributions to neural tube closure. Annu. Rev. Genet. 48, 583-611.

Yang, X.-J. and Seto, E. (2007). HATs and HDACs: from structure, function and regulation to novel strategies for therapy and prevention. Oncogene 26, 5310-5318.

Zhang, Q., Xue, P., Li, H., Bao, Y., Wu, L., Chang, S., Niu, B., Yang, F. and Zhang, T. (2013a). Histone modification mapping in human brain reveals aberrant expression of histone $\mathrm{H} 3$ lysine 79 dimethylation in neural tube defects. Neurobiol. Dis. 54, 404-413.

Zhang, Q., Xue, P., Li, H., Bao, Y., Wu, L., Chang, S., Niu, B., Yang, F. and Zhang, T. (2013b). Histone modification mapping in human brain reveals aberrant expression of histone $\mathrm{H} 3$ lysine 79 dimethylation in neural tube defects. Neurobiol. Dis. 54, 404-413.

Zhang, T., Cooper, S. and Brockdorff, N. (2015). The interplay of histone modifications - writers that read. EMBO Rep. 16, 1467-1481.

Zhang, W., Xia, X., Jalal, D. I., Kuncewicz, T., Xu, W., Lesage, G. D. and Kone, B. C. (2006). Aldosterone-sensitive repression of ENaCalpha transcription by a histone $\mathrm{H} 3$ lysine-79 methyltransferase. Am. J. Physiol. Cell Physiol. 290, C936-946.

Anmerkung: Englische Version des Artikels online verfügbar unter https://doi.org/10.1515/nf-2018-A001 


\section{Autoreninformationen}

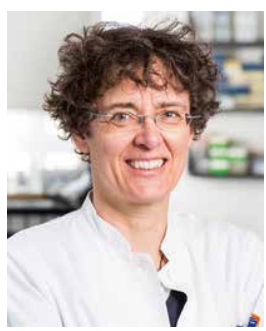

\section{Prof. Dr. Tanja Vogel}

Albert-Ludwigs-Universität Freiburg, Medizinische Fakultät, Institut für Anatomie und Zellbiologie, Abteilung für Molekulare Embryologie, Albertstr. 17, 79104 Freiburg, Deutschland

Tel.: +497612035086

E-Mail: Tanja.Vogel@anat.uni-freiburg.de

Prof. Dr. Tanja Vogel studierte an der Universität Hannover Biochemie (1994) und promovierte zum Dr. rer. nat. am Institut für Humangenetik an der Medizinischen Hochschule Hannover (1997). Ihre Zeit als Postdoktorandin verbrachte sie zunächst am MRC Human Genetics Unit in Edinburgh und anschließend am MPI für Biophysikalische Chemie in Göttingen. Ab 2005 war sie Gruppenleiterin in der Abteilung für Neuroanatomie am Zentrum für Anatomie der Universitätsmedizin Göttingen. Ihre Habilitation schloss sie im Jahre $2010 \mathrm{im}$ Fachbereich Anatomie ab. Für ihre Habilitation wurde ihr die Auszeichnung der besten Habilitation der Universitätsmedizin verliehen. Zum Sommersemester 2011 wechselte Frau Prof. Vogel an die Universität Freiburg. Seit 2014 ist sie kommissarische Leiterin der Abteilung für Molekulare Embryologie des Instituts für Anatomie und Zellbiologie.

Die Forschungsschwerpunkte von Frau Prof. Vogel liegen auf dem Gebiet der Neurobiologie: Epigenetische Histon-Modifikationen in ZNS-Entwicklung und Funktion, TGFß Signale der neuronalen Stammzelldifferenzierung und nicht-kodierende RNAs in neurologischen Entwicklungsstörungen.

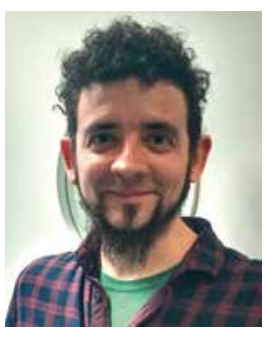

Alejandro Villarreal

Albert-Ludwigs-Universität Freiburg, Medizinische Fakultät, Institut für Anatomie und Zellbiologie, Abteilung für Molekulare Embryologie, Albertstr. 17, 79104 Freiburg, Deutschland

Tel.: +497612035108

E-Mail: Alejandro.Villarreal@anat.unifreiburg.de

Dr Alejandro Villarreal studierte Biologie an der Buenos Aires Universität. Er promovierte bis 2014 bei Dr. Alberto Javier Ramos mit einer Studie über reaktive Astrocyten in einem Schlaganfallmodell. Er ist Prof. Dr Vogel's Gruppe als Postdoktorand beigetreten, um Fachwissen im Forschungsbereich Epigenetik und Histonmodifikationen zu erwerben. Seine Forschungsinteressen liegen in den Bereichen Nervenzellendifferenzierung, in der Entwicklunsgbiologie und Gehirnschäden.

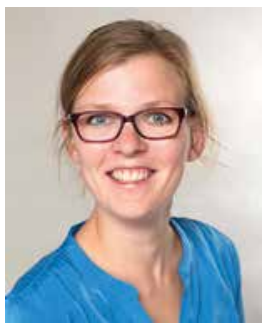
Dr. Henriette Franz
Albert-Ludwigs-Universität Freiburg, Medizinische Fakultät, Institut für Anatomie und Zellbiologie, Abteilung für Molekulare Embryologie, Albertstr. 17, 79104 Freiburg, Deutschland
Tel.: +497612035108
E-Mail: Henriette.Franz@anat.uni-freiburg. de

Frau Dr. Henriette Franz hat an der Universität Leipzig Biochemie (2005) studiert und am Max-Planck-Institut für biophysikalische Chemie in Göttingen zum Dr. rer. nat. (2010) promoviert. Als Postdoktorandin forschte sie zuerst am Zentrum für klinische Forschung der Uniklinik Freiburg (2015) und ist nun am Institut für Anatomie und Zellbiologie an der Universität Freiburg in der Lehre und in der Forschung tätig. Ein Schwerpunkt von Frau Dr. Franz ist die Neuroepigenetik. 